\title{
Thermomechanical Properties of the Coil of the Superconducting Magnets for the Large Hadron Collider
}

\author{
Karine Couturier, Paolo Ferracin, Walter Scandale, Ezio Todesco, and Davide Tommasini
}

\begin{abstract}
The correct definition and measurement of the thermomechanical properties of the superconducting cable used in high-field magnets is crucial to study and model the behavior of the magnet coil from assembly to the operational conditions. In this paper, the authors analyze the superconducting coil of the main dipoles for the Large Hadron Collider. They describe an experimental setup for measuring the elastic modulus at room and at liquid nitrogen temperature and for evaluating the thermal contraction coefficient. The coils exhibit strong nonlinear stressstrain behavior characterized by hysteresis phenomena, which decreases from warm to cold temperature, and a thermal contraction coefficient, which depends on the stress applied to the cable during cooldown.
\end{abstract}

Index Terms-Elastic modulus, mechanical properties, superconducting coil, thermal contraction coefficient.

\section{INTRODUCTION}

$\mathbf{T}$ HE STRESS-STRAIN relation of a superconducting coil and its thermal shrinkage plays a fundamental role in the structural behavior of superconducting magnets for particle accelerators [1], [2]. In particular, an optimal azimuthal prestress should be imposed to the coil during assembly and after cooldown, to prevent conductor motion during magnet excitation that may produce quenches limiting the maximum operational field. Moreover, coil stresses may cause displacements of cables with respect to the nominal design, thereby inducing intolerable field-shape errors, detrimental for the stability of circulating particles.

Thermomechanical analysis has been carried out for most of the modern superconducting magnets for accelerators [3]-[8]. The observed phenomena are rather unusual due to the composite structure of the involved material [9]-[13]. Indeed, it is well known that the stress-strain relation is not linear [14]-[16]. Moreover, the unloading phase is considerably different from the loading one and varies according to the maximum load reached in the compression phase (mechanical hysteresis). As the superconducting coil is subjected to a loading-unloading

Manuscript received June 24, 2001; revised January 7, 2002. The review of this paper was supervised by J. Schwartz.

K. Couturier is with the Surface and Material Technologies Group, EST Division, European Laboratory for Particle Physics (CERN), 1211 Geneva 23, Switzerland (e-mail: karine.couturier@ cern.ch).

P. Ferracin, W. Scandale, E. Todesco, and D. Tommasini are with the Main Magnets and Superconductors Group, LHC Division, European Laboratory for Particle Physics (CERN), 1211 Geneva 23, Switzerland (e-mail: paolo.ferracin@cern.ch; walter.scandale@cern.ch; ezio.todesco@cern.ch; davide.tommasini@cern.ch).

Publisher Item Identifier S 1051-8223(02)05430-1. cycle during magnet assembly, the final state of the coil at room temperature and its dimension [17], [18] is determined not only by its stress-strain relation, but also by the cycle used during the magnet assembly process.

The knowledge of the thermal contraction of the insulated cables [19], [20] is essential to explain why, during the cooldown, there is in general a nonnegligible loss of azimuthal prestress. This loss is typically induced by different thermal shrinkage between the coil and the collars, which surround and constrain the conductors. However, the measurement of the integrated thermal contraction coefficient of the coil is rather problematic. As stated in [21], "with such an ill-behaved material, a direct measurement of the thermal-contraction integral [...], at zero stress, is not very meaningful."

In this paper, we present measurements of the stress-strain relation of two types of superconducting insulated cables at 293 and at $77 \mathrm{~K}$. In particular, we focus on measurements of the stress-displacement curves along different loading and unloading paths, to obtain the data necessary to a complete modeling of the elasticity of the coil. By measuring the stress loss, we compute the integrated thermal contraction coefficient from 293 to $77 \mathrm{~K}$, at which most of the thermal contraction takes place. We explore the ambiguities in its definition, pointing out that different approaches chosen to evaluate the strains lead to significant variations in the obtained integrated thermal contraction. This also explains the rather large variation of thermal contraction values reported in the literature [21]-[26].

We analyze the superconducting coils (inner and outer layers) used in the main dipole of the Large Hadron Collider, the particle accelerator dedicated to high-energy physics under construction at European Organization for Nuclear Research (CERN). The LHC main dipole [27] consists of superconducting coils, clamped by austenitic steel collars and surrounded by an iron yoke, and a steel shrinking cylinder, all placed in a cryostat. It generates a magnetic field of $8.3 \mathrm{~T}$ at an operational temperature of $1.9 \mathrm{~K}$.

In this paper, we are not interested in measuring the intrinsic mechanical parameters of the individual coil components; instead, we define and evaluate the composite properties needed to describe the structural behavior of the magnet from assembly to cooldown.

In Section II of this paper, we describe the samples of superconducting cable which have been tested. The results of the stress-displacement measurements are given in Sections III and IV. Methods and data relative to the integrated thermal contraction coefficient are presented in Section V. Additional tests, cali- 
TABLE I

Basic PARAMETERS OF THE CABLES

\begin{tabular}{lcc}
\hline & Inner layer & Outer layer \\
\hline Number of strands & 28 & 36 \\
Cable width (mm) & 15.10 & 15.10 \\
Mid-thickness (mm) & 1.90 & 1.48 \\
Keystone angle (deg) & 1.25 & 0.90 \\
\hline
\end{tabular}

bration, and postprocessing procedures of experimental data are given in the Appendixes.

\section{SAMPLES}

The coil of the LHC dipole contains two layers (the so-called inner layer and outer layer) with two different keystoned Rutherford cables. The basic parameters of the cables are given in Table I. The cable insulation is polyimide and it is composed of two $50-\mu \mathrm{m}$ thick layers of butt lapped tape, each $11 \mathrm{~mm}$ wide and staggered by half the tape width, followed by a $69-\mu \mathrm{m}$ thick adhesive-coated tape 9-mm wide spiraling with 2-mm spacing to provide channels for helium penetration inside the coils [28].

We measured two conductor stacks of $15.4 \times 50 \mathrm{~mm}$, one (22 conductors) with the cable used for the internal layer, and one (28 conductors) for the external layer. The cables are stacked alternately in order to have a parallel-side sample. As for magnet coils, the samples are submitted to the following sizing-bounding cycle:

- heating for $30 \mathrm{~min}$ at $130{ }^{\circ} \mathrm{C}$ under a moderate stress (about $10 \mathrm{MPa}$ );

- compression to $80 \mathrm{MPa}$ and heating up to $185^{\circ} \mathrm{C}$ in a fixed volume for about $30 \mathrm{~min}$;

- cool down to room temperature in fixed volume.

\section{Elastic Modulus AT 293 K}

\section{A. Experimental Setup}

The stress-displacement curves have been measured using an electromechanical apparatus for high precision compression tests (UTS 200.4). The load is applied perpendicularly to the flat face of the conductors, which corresponds to the azimuthal direction in the dipole coil. No loads or constraints are applied in the direction parallel to the flat face of the cable. The precision of the cross-head motion for the displacement measurements is $1 \mu \mathrm{m}$, while the error on the values of the force is of $\pm 10 \mathrm{~N}$. The machine compresses the stacks with a constant cross-head speed of $0.05 \mathrm{~mm} / \mathrm{s}$; this value is similar to that used for clamping the dipole coils inside the collars [29]. A description of the calibration of the experimental apparatus using materials with known properties is given in Appendix I.

\section{B. Experimental Procedure and General Features}

In Figs. 1 and 2 we plot the stress versus the height of the conductor stacks (inner layer and outer layer, respectively) measured by the electromechanical apparatus. The compression force is increased until a peak value, then reduced to zero. We performed five different loading cycles with decreasing peak stresses, respectively, 127, 111, 95, 79, and $63 \mathrm{MPa}$. After

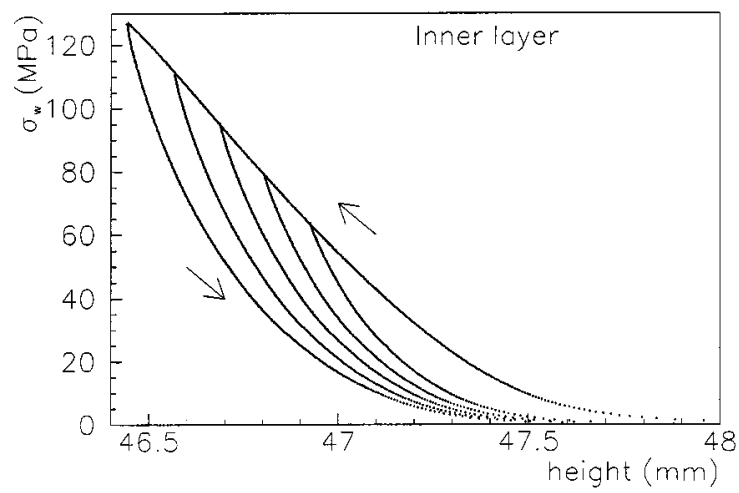

Fig. 1. Stress $\sigma_{w},(\mathrm{MPa})$ at $293 \mathrm{~K}$ versus total height $l_{w}$ (mm) for the inner layer conductor stack, loading and unloading curves from different peak stresses (experimental data).

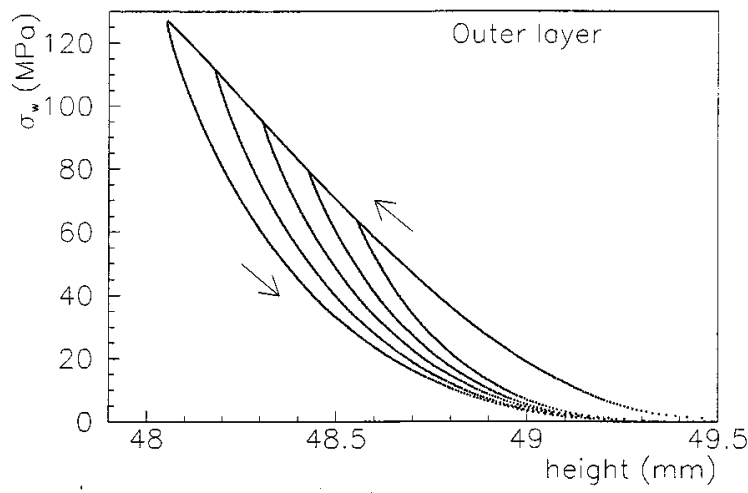

Fig. 2. Stress $\sigma_{w}(\mathrm{MPa})$ at $293 \mathrm{~K}$ versus total height $l_{w}(\mathrm{~mm})$ for the outer layer conductor stack, loading and unloading curves from different peak stresses (experimental data).

each cycle, when the stress on the stack is reduced to zero, the contact between the stack and the flat-plate fixtures of the electromechanical apparatus is lost. This allows the stack to relax completely. The loading curve has a good reproducibility if the stack is completely unloaded at the end of a cycle. In Appendix II we present the behavior of the samples when the compression cycles start from different stress values.

As is well known, the stacks feature a different behavior in the loading and in the unloading branches. The loading phase is characterized by a smaller slope. The cycle shows a hysteresis, which depends on the peak stress reached during the compressive phase. The stress-displacement curves are nonlinear, both in the loading and in the unloading phases. The stack rigidity increases with stress.

\section{Definition of Elastic Modulus}

At room temperature, the elastic modulus $E_{w}$ is given by

$$
E_{w}=\frac{d \sigma_{w}}{d \epsilon_{w}}=\frac{d \sigma_{w}}{d l_{w}} l_{w 0}
$$

where $l_{w 0}$ is the unloaded stack height and $\sigma_{w}$ is the stress measured at ambient conditions. The stack is very soft at low stresses, where small stress changes give rise to very large displacements (see Figs. 1 and 2). Following [21] and [22], we define $l_{w 0}$ as the stack height for a small stress $(0.4 \mathrm{MPa})$ on the loading curve. We evaluate this parameter as the average of 


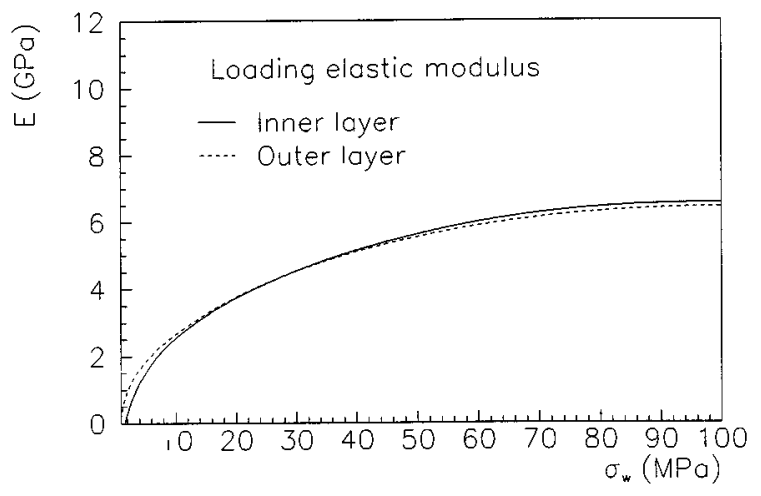

Fig. 3. Elastic modulus $E(\mathrm{GPa})$ at $293 \mathrm{~K}$ versus stress $\sigma_{w}(\mathrm{MPa})$ for the conductor stacks, loading curves.

the stack height over 15 different cycles and we assign a statistical error of $2 \times$ rms observed over the 15 cycles (giving a $95 \%$ confidence level). We obtain an unloaded height of 48.16 \pm 0.14 for the inner layer stack and $49.51 \pm 0.08$ for the outer layer stack. This relative error of 0.2 to $0.3 \%$ has practically no impact on the computation of the elastic modulus according to (1). To evaluate the tangent of the stress-displacement curves, we fitted experimental measurements with a fifth-order polynomial and we analytically computed its derivative. This method provides more precise results compared to a direct evaluation of the derivative.

\section{Results}

In Fig. 3, we plot the elastic modulus of the inner and outer stacks computed by the stress-displacement loading curves at $293 \mathrm{~K}$. Each value has a reproducibility within a range of $\pm 7 \%$ (corresponding to $\pm 2 \times \mathrm{rms}$ ). The elastic modulus features a large increase (from 0 to $6 \mathrm{GPa}$ ) up to a stress of $70 \mathrm{MPa}$, and then it remains almost constant for higher stresses (from $70 \mathrm{MPa}$ up to at least $100 \mathrm{MPa}$ ). No significant differences are observed between the two layers.

The elastic modulus during the unloading phase is much larger than the one of the loading phase and it features an almost linear dependence on the stress, as already observed in [30]; but, as shown in Figs. 4 and 5 it depends on the peak stress. For the same load, the higher the peak stress of the cycle, the smaller the elastic modulus. For instance, at $70 \mathrm{MPa}$, which is the nominal stress acting azimuthally on the coil at the end of the magnet assembly, the elastic modulus varies from 11 to $14 \mathrm{GPa}$ for the inner coil and from 10 to $13 \mathrm{GPa}$ for the outer coil for a peak stress of the compression cycle varying between 80 and $130 \mathrm{MPa}$. Each value plotted in Figs. 4 and 5 has a reproducibility within a range of $\pm 5 \%$ (corresponding to $\pm 2 \times \mathrm{rms})$.

\section{Elastic Modulus at $77 \mathrm{~K}$}

\section{A. Experimental Setup, Procedure, and General Features}

The measurements at $77 \mathrm{~K}$ have been performed in a cryostat filled with liquid nitrogen. Results obtained on materials with known properties are given in Appendix I. Also in this case, we performed five different loading cycles with decreasing peak

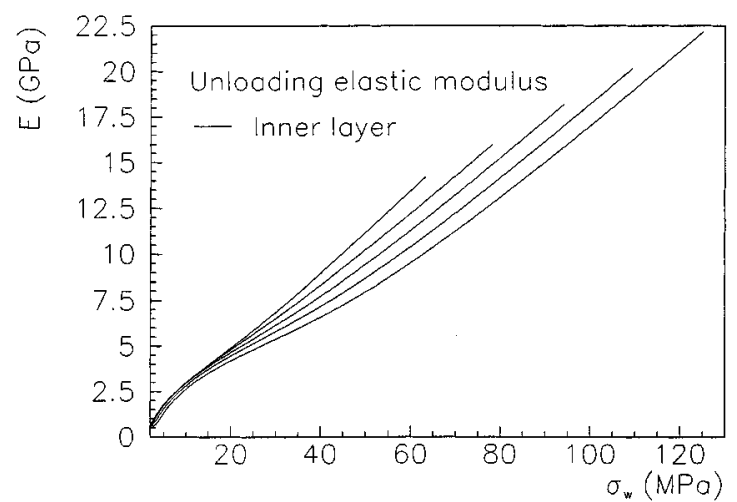

Fig. 4. Elastic modulus $E(\mathrm{GPa})$ at $293 \mathrm{~K}$ versus stress $\sigma_{w}(\mathrm{MPa})$ for the inner layer conductor stack, unloading curve.

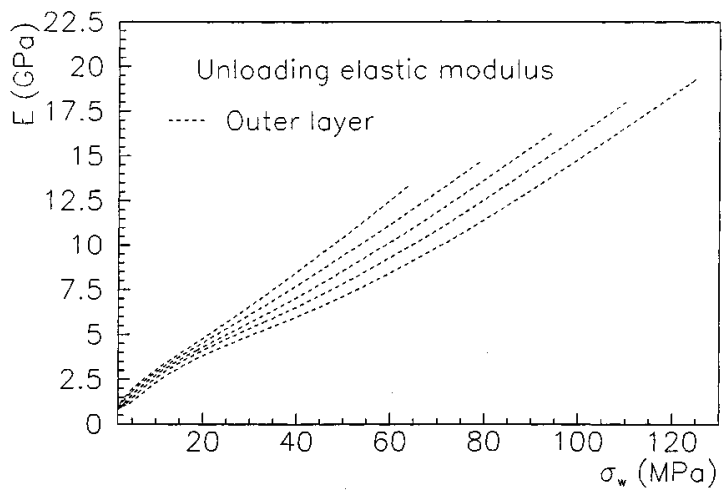

Fig. 5. Elastic modulus $E(\mathrm{GPa})$ at $293 \mathrm{~K}$ versus stress $\sigma_{w}(\mathrm{MPa})$ for the outer layer conductor stack, unloading curve.

stresses, respectively, 100, 75, 60, 45, and 30 MPa. In Figs. 6 and 7 we show the stress-displacement curves obtained at liquid nitrogen temperature for the inner and outer layer, respectively. The curves feature two main differences with respect to measurements at $293 \mathrm{~K}$. First, the loading branch of the cycle is characterized by steeper slope than at room temperature, and secondly the hysteresis between the loading and the unloading curves is considerably smaller.

\section{B. Definition of Elastic Modulus}

In order to compute the elastic modulus of the stacks, also at $77 \mathrm{~K}$, it is necessary to define an unloaded height. The elastic modulus is defined as in (1), that is

$$
E_{c}=\frac{d \sigma_{c}}{d \epsilon_{c}}=\frac{d \sigma_{c}}{d l_{c}} l_{c 0}
$$

where $E_{c}, \sigma_{c}$, and $l_{c 0}$ are, respectively, the elastic modulus, the stress, and the unloaded stack height at liquid nitrogen temperature. Since our experimental apparatus for the stress-displacement measurements provides at nitrogen temperature only relative displacements and not absolute values of the stack height, we assume for the numerical evaluation of the elastic modulus at $77 \mathrm{~K}$ that $l_{c 0}=l_{w 0}$. Indeed, the difference between $l_{c 0}$ and $l_{w 0}$, that is the integrated thermal contraction, is of the order of $1 \%$. Therefore, by assuming $l_{c 0}=l_{w 0}$ in the computation of $E_{c}$, we introduce an error of $1 \%$ which is negligible for our purposes. 


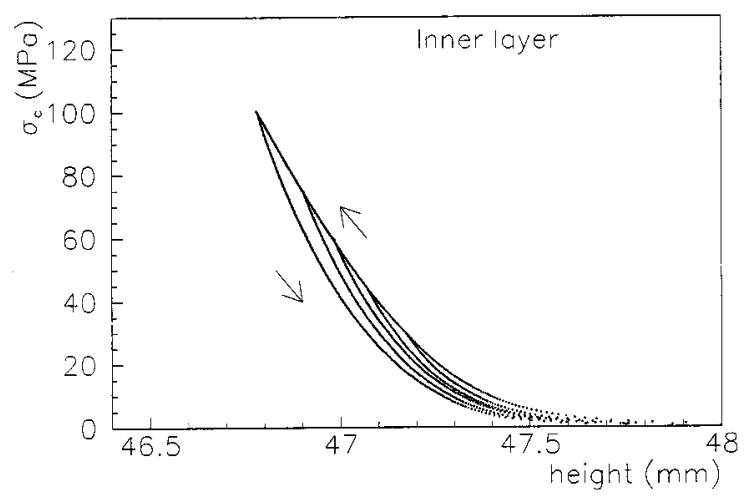

Fig. 6. Stress $\sigma_{c}(\mathrm{MPa})$ at $77 \mathrm{~K}$ versus total height $l_{c}(\mathrm{~mm})$ for the inner layer conductor stack, loading and unloading curves from different peak stresses (experimental data).

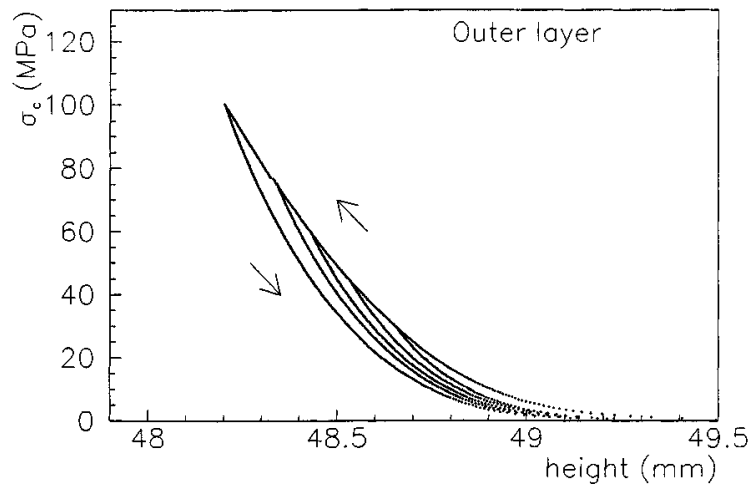

Fig. 7. Stress $\sigma_{c}(\mathrm{MPa})$ at $77 \mathrm{~K}$ versus total height $l_{c}(\mathrm{~mm})$ for the outer layer conductor stack, loading and unloading curves from different peak stress (experimental data).

\section{Results}

The elastic modulus computed for the loading curve at $77 \mathrm{~K}$ (see Fig. 8) is about 50\% higher than the value at ambient temperature for stresses higher than $70 \mathrm{MPa}$. On the other hand, in the unloading phase (see Figs. 9 and 10) the elastic moduli evaluated at $77 \mathrm{~K}$ are very similar to the ones at ambient temperature, but with a less pronounced dependence on the peak stress of the cycle. Also in this case, each value has a reproducibility within a range of $\pm 5 \%$ (corresponding to $\pm 2 \times$ rms).

\section{INTEGRATED THERMAL CONTRACTION}

\section{A. Measurement Method}

The integrated thermal contraction coefficient is defined as the relative difference of the unloaded heights between two temperatures [31], i.e.,

$$
\alpha=\frac{l_{w 0}-l_{c 0}}{l_{w 0}} .
$$

If one could measure the unloaded height at both temperatures, one could perform a direct estimate of $\alpha$. In Section III we pointed out that the unloaded height of the stack is ill defined; in fact, small variations of the load, say from 0 to $1 \mathrm{MPa}$, induce a large variation of the stack height of the order of $0.5 \mathrm{~mm}$ (see Figs. 1 and 2), i.e., 1\% of our sample height. This is the same order of magnitude of the integrated thermal contraction

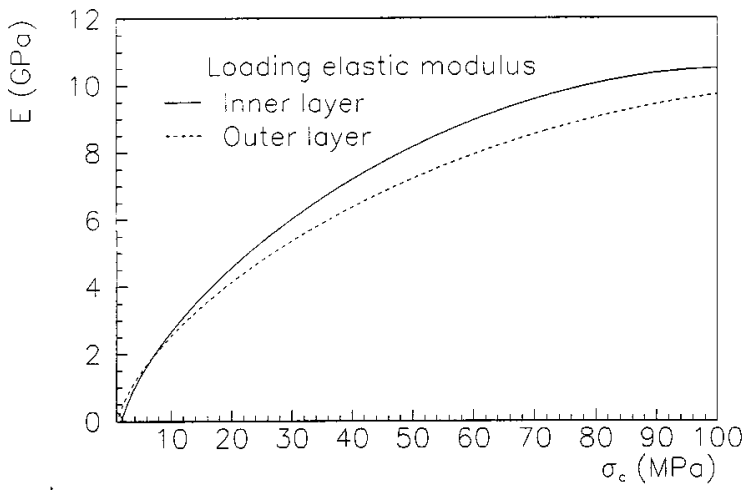

Fig. 8. Elastic modulus $E(\mathrm{GPa})$ at $77 \mathrm{~K}$ versus stress $\sigma_{c}(\mathrm{MPa})$ for the conductor stacks, loading curve.

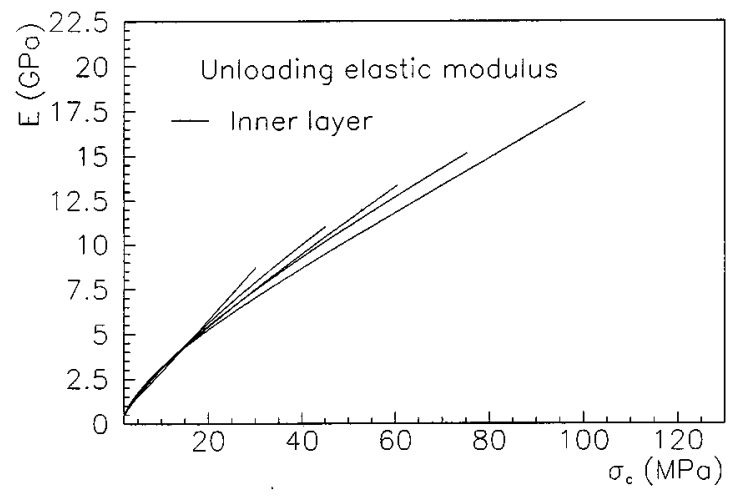

Fig. 9. Elastic modulus $E(\mathrm{GPa})$ at $77 \mathrm{~K}$ versus stress $\sigma_{c}(\mathrm{MPa})$ for the inner layer conductor stack, unloading curve.

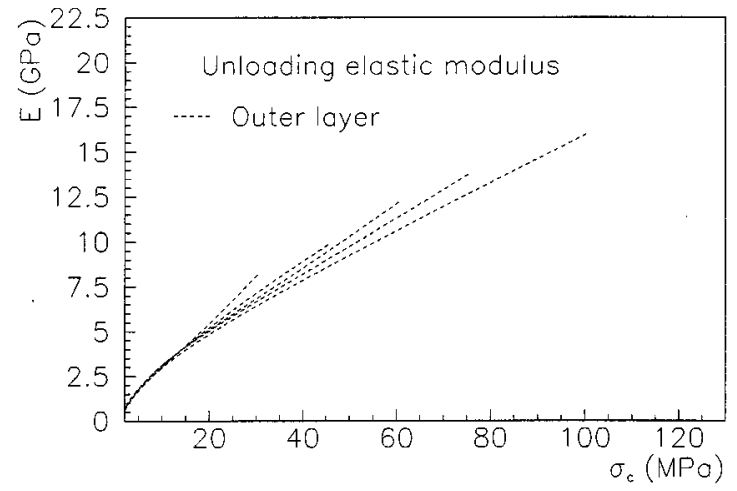

Fig. 10. Elastic modulus $E(\mathrm{GPa})$ at $77 \mathrm{~K}$ versus stress $\sigma_{c}(\mathrm{MPa})$ for the outer layer conductor stack, unloading curve.

of the stack [21]-[26]. We also showed that, with a low stress of about $0.4 \mathrm{MPa}$, the stack height can be determined with a relative precision of about $0.3 \%$. This indetermination as well is comparable to the thermal shrinkage that we want to measure. Therefore, a direct measure of this quantity using (3) is not possible.

The thermal contraction can be also evaluated using the stress loss in a fixed cavity (see for instance [22]) and the stress-strain relation of the samples. In an infinitely rigid cavity of known integral thermal contraction coefficient $\alpha_{f}$, the mechanical deformation of the sample is equal to the difference in thermal contraction

$$
\epsilon_{w}-\epsilon_{c}=\alpha_{s}-\alpha_{f}
$$


where $\alpha_{s}$ is the unknown thermal coefficient of the sample and $\epsilon_{w}$ and $\epsilon_{c}$ the deformations at ambient and cryogenic temperature. If the sample has a linear elastic behavior, that is

$$
\epsilon_{w}=\frac{\sigma_{w}}{E_{w s}} \quad \epsilon_{c}=\frac{\sigma_{c}}{E_{c s}}
$$

one has

$$
\alpha_{s}=\frac{\sigma_{w}}{E_{w s}}-\frac{\sigma_{c}}{E_{c s}}+\alpha_{f} .
$$

In our case, we have a nonlinear behavior and therefore strain dependence on the stress has to be evaluated using experimental data. Equation (6) can be generalized to include also the deformations of the mould. Let $E_{w f}$ and $E_{c f}$ be the elastic moduli of the cavity at warm and cold temperature, respectively. Then, one has

$$
\alpha_{s}=\epsilon_{w}\left(\sigma_{w}\right)+\frac{\sigma_{w}}{E_{w f}}-\epsilon_{c}\left(\sigma_{c}\right)-\frac{\sigma_{c}}{E_{c f}}+\alpha_{f} .
$$

To deduce the thermal contraction from the measurement of the stress loss in a fixed cavity by (7), one must know strains; therefore, also in this case, one has to make assumptions on the unloaded coil height. As already mentioned in the Section IV-B, an uncertainty on the unloaded stack height has very little influence in the definition of the elastic modulus, leading in our case to a maximum error of the order of $1 \%$. On the other hand, different assumptions on the unloaded stack height lead to very large differences in the strains values $\epsilon_{w}\left(\sigma_{w}\right)$ and $\epsilon_{c}\left(\sigma_{c}\right)$ of (7) and consequently in $\alpha_{s}$. Moreover, strains vary significantly according to the $\sigma-\epsilon$ relation assumed for the stacks. If we consider a linear behavior, that is an elastic modulus independent on stress (see for instance [21], [22]), we will obtain a much lower strain value with respect to the case with the complete curve and therefore a different $\alpha_{s}$. Finally, the hysteresis of the stress-strain curve at $77 \mathrm{~K}$ yields a further uncertainty to the problem. In fact, it is not clear how to evaluate $\epsilon_{c}\left(\sigma_{c}\right)$ in (7), since it is not known which curve is reached by the stacks at the end of the cooldown (loading or unloading).

One must conclude that the integral thermal contraction of a stack of film-insulated cables is not uniquely defined, as it depends on the scheme used to derive the strain from the displacements. Here, we propose the following scheme.

- We use the experimental nonlinear stress-displacement relation to evaluate the strain from the stress

$$
\epsilon_{w s}=\frac{\Delta l_{w}}{l_{w 0}} \quad \epsilon_{c s}=\frac{\Delta l_{c}}{l_{c 0}} .
$$

The effect of a linear stress-displacement relation on the evaluation of the strains and of the thermal contraction is given in Appendix V.

- We fix the unloaded coil height $l_{w 0}$ and $l_{c 0}$ as the height of the stack measured with the electromechanical apparatus at a stress of $0.4 \mathrm{MPa}$ on the loading curve, as already explained in Sections III and IV. The influence of the cutstress on the evaluation of the strains and of the thermal contraction is given in Appendix IV.

- A $77 \mathrm{~K}$, we compute the strain after the cooldown $\epsilon_{c}\left(\sigma_{c}\right)$ both on the loading curve and on the unloading curve. In the latter path, we assume a peak stress $\hat{\sigma_{c}}=\sigma_{w}$. In this way we obtain two estimates of the integrated thermal contraction coefficient. The first describes the ideal case of a cooldown in absence of stress followed by the application of the stress up to $\sigma_{c}$. The second path instead describes another ideal case with a cooldown at constant stress $\sigma_{w}$ followed by a reduction of the stress from $\sigma_{w}$ to $\sigma_{c}$. Analyzing these two extreme cases, the obtained range of variation of the thermal contraction coefficient can be considered as an uncertainty associated to the hysteresis at $77 \mathrm{~K}$.

\section{B. Experimental Setup}

We use a carbon steel mould: the stress is applied by a screw, placed at the top of the mould, and is measured by two capacitive gauges [32], placed at the top and the bottom of the stack. The sensitivity of the capacitive gauges is $1 \mathrm{MPa}$. In order to measure the stress loss from warm to cold temperature, the device is submerged in liquid nitrogen in a cryostat. The integral thermal contraction coefficient from $293 \mathrm{~K}$ to $77 \mathrm{~K}$ is computed through (7) by the measurement of the stress loss $\left(\sigma_{w}-\sigma_{c}\right)$ in the cavity. Tests of the apparatus on materials with known properties are given in Appendix I.

\section{Experimental Procedure and Results}

We performed five different cooldowns with two stacks $100 \mathrm{~mm}$ high, one composed with inner layer conductors and one with outer layer conductors. At $293 \mathrm{~K}$, the stacks have been loaded to the peak stress and then unloaded to a value of stress $\sigma_{w}$ equal to the $60 \%$ of the peak stress. This procedure has been chosen taking into account the loading path followed by the coil during the magnet assembly before the cooldown [33]. For example, in the first measurement the stack has been loaded to $127 \mathrm{MPa}$ and then unloaded to $80 \mathrm{MPa}$.

The strain $\epsilon_{w s}$ has been computed as the difference between the stack height at $0.4 \mathrm{MPa}$ on the loading curve and the height on the unloading curve at a stress of $80 \mathrm{MPa}$. At cryogenic temperature the strain $\epsilon_{c s}$ can be computed in the same way. Two estimates are computed: the first one $\left(\alpha_{l}\right)$ by assuming that at $77 \mathrm{~K}$ the stack follows the loading curve, the second one $\left(\alpha_{u}\right)$ by considering that it follows the unloading curve whose peak stress at $77 \mathrm{~K}$ is $\sigma_{w}$.

In Figs. 11 and 12, we present the measured stress losses for the inner and outer layer, while in Figs. 13 and 14 we plot the average values between $\alpha_{l}$ and $\alpha_{u}$, with the bar indicating the range of variation between these two case. We can see that the difference between $\alpha_{l}$ and $\alpha_{u}$ is not negligible, i.e., around $10^{-3}$. Indeed, the dependence of $\alpha$ on the stress $\sigma_{w}$ is much stronger. One observes a variation from $0.006-0.007$ for $\sigma_{w}=$ $40 \mathrm{MPa}$ up to $0.009-0.012$ for $\sigma_{w}=80 \mathrm{MPa}$.

In order to check if the integral thermal contraction coefficient of the coil depends only on the stress at $293 \mathrm{~K}$, but also on the stress at $77 \mathrm{~K}$, we made additional measurements on the outer layer stack, cooling down from the same stresses when warm and changing the stresses when cold by modifying the thermal shrinkage of the mould. This can be realized by replacing half of the stack with a material with a known thermal shrinkage, different from that of the press. We used either aluminum or invar. In the first case the cavity has a lower thermal shrinkage, and the stress loss is increased; in the second case 


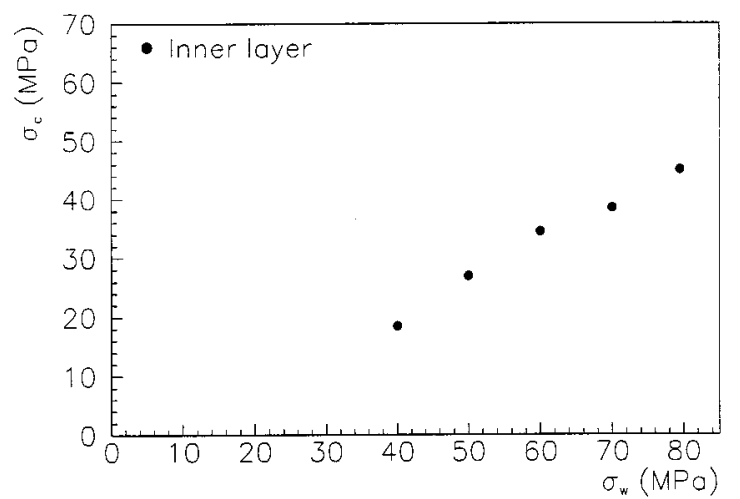

Fig. 11. Stress $\sigma_{c}(\mathrm{MPa})$ at $77 \mathrm{~K}$ versus stress $\sigma_{w}(\mathrm{MPa})$ at $293 \mathrm{~K}$ for the inner layer stack.

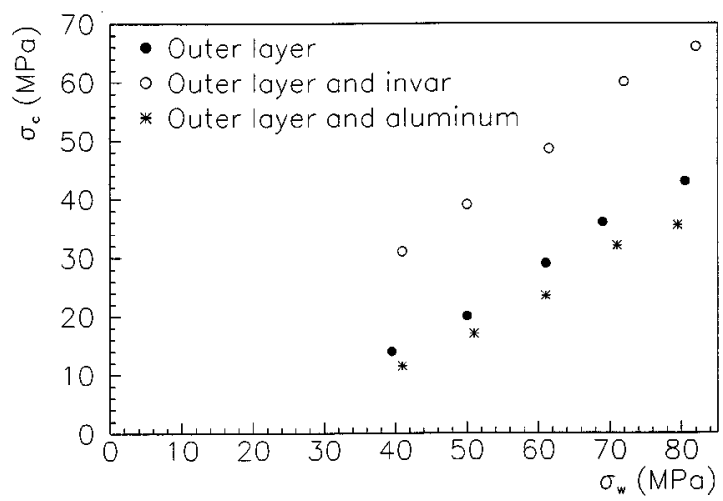

Fig. 12. Stress $\sigma_{c}(\mathrm{MPa})$ at $77 \mathrm{~K}$ versus stress $\sigma_{w}(\mathrm{MPa})$ at $293 \mathrm{~K}$ for the outer layer stack, with different equivalent thermal shrinkage of the mould.

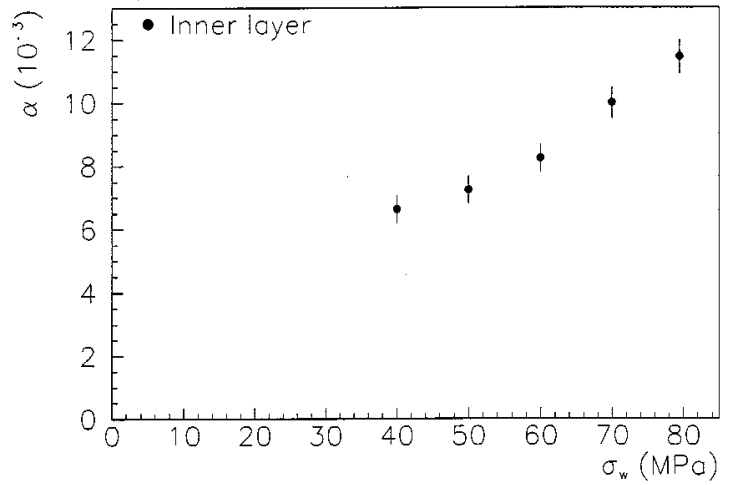

Fig. 13. Integrated thermal contraction coefficient $\alpha$ versus stress $\sigma_{w}$ (MPa) at $293 \mathrm{~K}$ for the inner layer stack.

one has the opposite situation. Explicit computations are given in Appendix III. In Figs. 12 and 14 the results are shown. No significant difference is found between the case of the full stack and of the stack with aluminum, whose stress losses differ by less than $10 \mathrm{MPa}$. A decrease in the thermal contraction coefficient of around 0.002 is found for the cavity with invar, where the stress loss is reduced by $15-20 \mathrm{MPa}$. This decrease is approximately constant for the five considered cases, leading to an offset between the two $\sigma_{w}-\alpha$ curves. One can conclude that the integrated thermal contraction of a stack of film-insulated cable depends on the stress path followed during the cooldown.

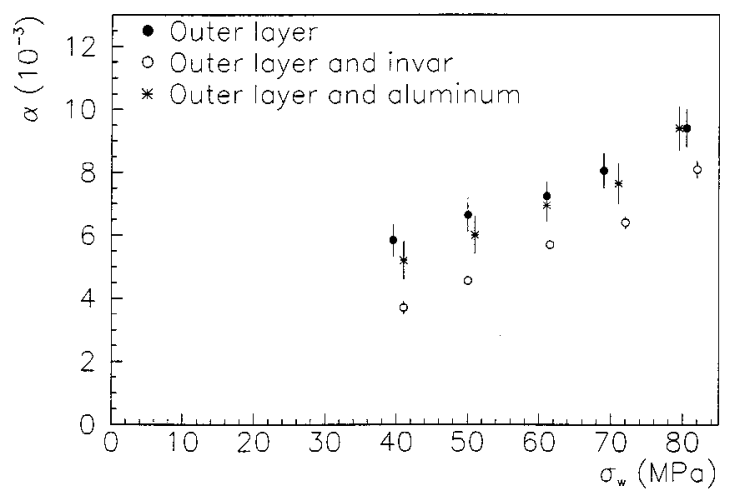

Fig. 14. Integrated thermal contraction coefficient $\alpha$ versus stress $\sigma_{w}(\mathrm{MPa})$ at $293 \mathrm{~K}$ for the outer layer stack, with different equivalent thermal shrinkage of the mould.

\section{CONCLUSION}

We presented the results of measurements of the thermomechanical properties of rectangular stacks of insulated cables of the LHC dipole coil at $293 \mathrm{~K}$ and $77 \mathrm{~K}$. We measured stress-displacement curves along different paths that are followed during the magnet assembly and cooldown.

The stress-displacement relations are nonlinear, and elastic moduli are defined as the derivatives of these curves. At $293 \mathrm{~K}$, the elastic modulus in the loading phase is around $6 \mathrm{GPa}$ for stresses above $70 \mathrm{MPa}$ and goes to zero for decreasing loads. At $77 \mathrm{~K}$, a $50 \%$ increase of the elastic modulus is observed in the loading phase. In the unloading phases, elastic moduli are the same at $293 \mathrm{~K}$ and at $77 \mathrm{~K}$; they feature a roughly linear dependence on the stress.

We pointed out severe difficulties in defining strains, due to the lack of precision of the unloaded coil height. This has no effect on the evaluation of the elastic modulus but strongly affects the measurement of the thermal contraction coefficient. Strains depend on the cut-stress used to define the unloaded coil height, on the scheme used to model the stress-displacement curve (linear or nonlinear), and, at $77 \mathrm{~K}$, on the branch of the curve reached after cooldown (loading or unloading).

We evaluated the thermal contraction coefficient measuring stress loss from five different stresses at $293 \mathrm{~K}$ and different cooldown configurations, obtaining results that range from 0.004 to 0.011 . Different assumptions on the trajectory in the $(\epsilon, \sigma)$ plane followed during cooldown (either along loading or unloading curve) lead to additional variations of around 0.001. We showed that different cut-stresses and linear or nonlinear approximation for the stress-strain relation also lead to significant variations in the thermal contraction coefficient derived from stress loss.

We conclude that the thermal contraction coefficient significantly depends on the scheme used to derive it from measurements. This explains the rather wide range of results that can be found in the literature. In fact, the thermal contraction is used to correctly model prestress loss from room to cryogenic temperature in the superconducting coils. For this reason, the choice of the convention for determining strains is not decisive, i.e., using linear or nonlinear approximation and choosing arbitrarily the minimum stress the "unloaded" coil length. One can choose any convention, provided that inconsistencies are avoided. If a linear 
TABLE II

Measurements of the Sample Elastic Modulus (GPa) AT 293 K AND 77 K AND COMPARISON WITH REFERENCE VALUES

\begin{tabular}{lcc}
\hline $293 \mathrm{~K}$ & Measure & Reference \\
\hline Aluminium & $73 \pm 1$ & 70 \\
Copper & $125 \pm 1$ & 120 \\
Invar & $151 \pm 1$ & 150 \\
Steel & $200 \pm 8$ & 195 \\
\hline \hline $77 \mathrm{~K}$ & Measure & Reference \\
\hline Aluminium & $78 \pm 3$ & 77 \\
Copper & $128 \pm 6$ & 138 \\
Invar & $129 \pm 9$ & 150 \\
Steel & $206 \pm 15$ & 209 \\
\hline
\end{tabular}

TABLE III

INTEGRAL THERMAL CONTRACTION COEFFICIENT $\alpha\left(10^{-3}\right)$ FrOM $293 \mathrm{~K}$ TO 77 K EVALUATED BY THE STRESS LOSSES AND COMPARISON WITH REFERENCE VALUES

\begin{tabular}{lcc}
\hline & Meas. & Reference \\
\hline Aluminium & $3.9 \pm 0.3$ & 3.9 \\
Invar & $0.4 \pm 0.1$ & 0.5 \\
Copper & $3.2 \pm 0.3$ & 3.0 \\
\hline
\end{tabular}

elasticity modulus is used, thermal contraction must be evaluated with strains defined in the linear approximation. Equivalently, the same cut-stress must be used to define strains and to evaluate the thermal contraction.

\section{APPENDIX I}

\section{CALIBRATION OF EXPERIMENTAL APPARATUS}

The measurements of the elastic modulus at 293 and 77 K performed by the electromechanical apparatus have been checked with four 100-mm high metallic samples (aluminum 6082 T6, copper, invar, and steel $316 \mathrm{LN}$ ). No measurable hysteresis has been observed. In Table II, we give the results of the measurements with an error of two sigmas. A good agreement with the reference values is found.

Thermal contraction coefficient measurements have been calibrated by measuring stress losses of three different materials (aluminum 6082 T6, invar, and copper) in our mould. In Table III, we show the measurement results that are in good agreement with the literature.

\section{APPENDIX II DIFFERENT LOADING PROCEDURES}

We performed two tests to analyze the behavior of the stack in different conditions with respect to the measurements presented in the previous sections. First, we compressed the stack with partial unloadings (see Fig. 15). This is the procedure used during the collaring of the coil. One observes that after partial unloading, during the reloading cycle the stack follows the unloading curve, but once it reaches the maximum stress of the previous loading phase, it deforms along the initial loading curve. The total deformation at the end of the compression phase is given by the monotonic loading curve. This phenomenon has been observed also for the impregnated $\mathrm{Nb}_{3} \mathrm{Sn}$ composites [34], [35].

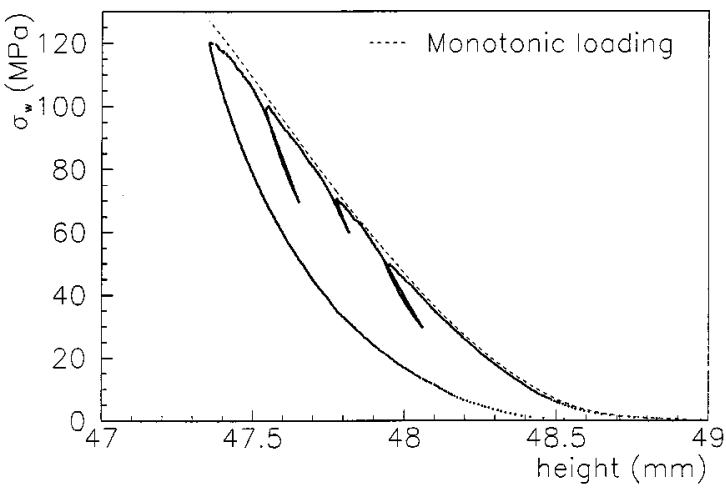

Fig. 15. Stress $\sigma_{w}$ (MPa) at $293 \mathrm{~K}$ versus total height $l_{w}$ (mm) for the outer layer conductor stack, loading with three steps: experimental data and comparison with the monotonic loading curve.

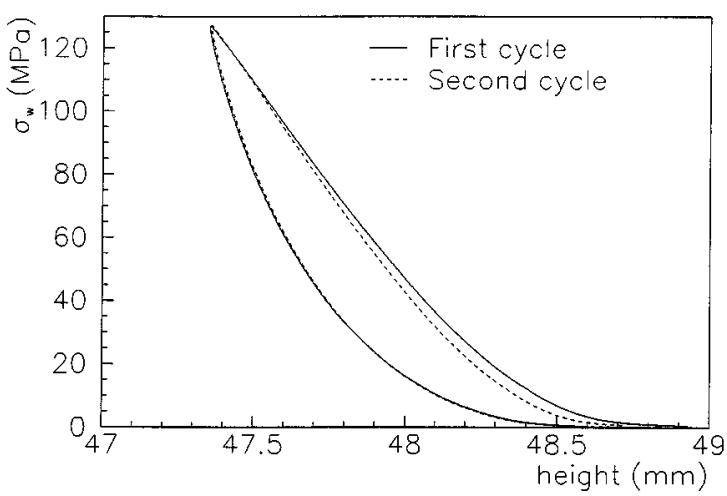

Fig. 16. Stress $\sigma_{w}(\mathrm{MPa})$ at $293 \mathrm{~K}$ versus total height $l_{w}(\mathrm{~mm})$ for the outer layer conductor stack: experimental data of two consecutive cycles.

In Fig. 16, we show two consecutive cycles: the first one starts from a completely unloading condition (as all cycles analyzed in the main text of this paper), and the second one is performed after the first cycle without a complete removal of the stress. The stack shows some memory of the deformation of the first cycle during the loading curve, which has a slightly higher slope.

\section{APPENDIX III \\ MODIFICATIONS OF THERMAL SHRINKAGE OF THE MOULD}

We changed the integral thermal contraction of the carbon steel mould by inserting a filler with a different shrinkage with respect to the carbon steel. This is equivalent to cooldown, the stack in a mould with a different thermal contraction coefficient, with a consequent variation of the stress losses. In particular, in this case (7) becomes

$$
\alpha_{s}=\epsilon_{w s}+\frac{\sigma_{w}}{E_{w e q}}-\epsilon_{c s}-\frac{\sigma_{w}}{E_{c e q}}+\alpha_{e q}
$$

where

$$
\begin{gathered}
\frac{1}{E_{w e q}}=\frac{R+1}{E_{w f}}+\frac{1}{E_{w m}} \\
\frac{1}{E_{c e q}}=\frac{R+1}{E_{c f}}+\frac{1}{E_{c m}}
\end{gathered}
$$


TABLE IV

STRAINS (IN $10^{-3}$ UNITS) AND INTEGRAL THERMAL CONTRACTION COEFFICIENT $\alpha$ (in $10^{-3}$ UNITS) OF THE INNER LAYER EVALUATED FROM THREE DiFFERENT CUT-STRESSES (MPa)

\begin{tabular}{cccccc}
\hline \multicolumn{7}{l}{ Inner layer } \\
\hline$\sigma_{w}$ & $\sigma_{c}$ & cut-stress & $\epsilon_{w}$ & $\epsilon_{c l}$ & $\alpha_{l}$ \\
\hline 80 & 45 & 0.4 & 35.9 & 26.0 & 12.0 \\
80 & 45 & 1.0 & 28.9 & 17.7 & 13.2 \\
80 & 45 & 3.0 & 23.3 & 11.6 & 14.0 \\
\hline
\end{tabular}

TABLE V

STRAINS (IN $10^{-3}$ UNITS) AND INTEGRAL THERMAL CONTRACTION COEFFICIENT $\alpha$ (IN $10^{-3}$ UNITS) OF THE INNER LAYER EVALUATED WITH DIFFERENT $\sigma-\epsilon$ RELATION (MPa)

\begin{tabular}{cccccc}
\hline \multicolumn{7}{l}{ Inner layer } \\
\hline$\sigma_{w}$ & $\sigma_{c}$ & $\sigma-\epsilon$ relation & $\epsilon_{w}$ & $\epsilon_{c u}$ & $\alpha_{u}$ \\
\hline 80 & 45 & non-linear & 35.9 & 27.0 & 10.9 \\
80 & 45 & linear & 6.2 & 4.8 & 3.4 \\
\hline
\end{tabular}

and

$$
\alpha_{e q}=\alpha_{f}-R\left(\alpha_{m}-\alpha_{f}\right) .
$$

In (10)-(12) $R$ is the ratio between the height of the filler and the height of the stack, and $E_{w m}, E_{c m}$, and $\alpha_{m}$ are, respectively, the elastic modulus at room temperature, the elastic modulus at 77 $\mathrm{K}$, and the integral thermal contraction coefficient of the filler.

\section{APPENDIX IV \\ DEPENDENCE OF THE INTEGRATED THERMAL CONTRACTION ON THE CUT-STRESS}

We analyzed the effect of the stress chosen to define the unloaded stack height on the strains and on the integrated thermal contraction coefficient measured with stress loss method. We define $l_{w 0}$ and $l_{c 0}$ as the height of the stack at three different cut-stresses $0.4,1.0$, and 3.0 MPa. We then evaluate the strain of the stack at 293 and $77 \mathrm{~K}$ on the loading curve, and we compute the thermal contraction. In Table IV, we can see that strains strongly depend on the cut-stress (up to a factor two). Integral thermal contraction also shows significant variations (about $15 \%$ with respect to the analyzed range of cut-stresses).

\section{APPENDIX V \\ INTEGRATED THERMAL CONTRACTION OF THE STACK WITH A LINEAR STRESS-STRAIN RELATION}

Here, we study the impact of the stress-strain modeling of the stack on the integral thermal contraction. We consider a linear behavior and we compute the strain before and after the cooldown assuming a constant elastic modulus. We assume that the strain at $75 \mathrm{MPa}$ at room temperature is given by stress divided by the elastic modulus of the unloaded curve (12900 MPa for the inner layer). We performed the same computation at cryogenic temperature considering the elastic modulus of the unloading curve at $45 \mathrm{MPa}$ (9400 $\mathrm{MPa}$ for the inner layer) and we compare the results with the approach followed in the main text. The aim of this analysis is to evaluate what happens if we assume a linear stress-strain relation instead of the nonlinear one. One finds a very large difference between the two values (see Table V).

\section{ACKNOWLEDGMENT}

The authors would like to thank S. Sgobba for useful comments and suggestions on elastic modulus measurements. They wish to acknowledge S. Caspi for discussing about the coil thermal contraction coefficient and A. Devred for several discussions on the mechanical properties of superconducting cables as well as the the problem of the prestress loss in superconducting magnets.

\section{REFERENCES}

[1] K.-H. Mess, P. Schmuser, and S. Wolff, Superconducting Accelerator Magnets. Singapore: World Scientific, 1996.

[2] M. N. Wilson, Superconducting Magnets. Oxford, U.K.: Clarendon, 1986.

[3] M. S. Chapman, "A finite element analysis of an SSC dipole magnet (NC-9 cross-section)," in Supercollider 1, M. McAshan, Ed. New York: Plenum, 1989, pp. 659-668.

[4] M. Bona, M. Modena, and D. Perini, "Finite element applications to the design of high-energy physics equipment," in Proc. ANSYS Fifth Int. Conf. Exhibition, D. E. Dietrich, Ed. Pittsburgh, PA, 1991, p. 17.30.

[5] M. Bona and D. Perini, "Finite-element structural analysis of the $10 \mathrm{~m}$ long dipole prototype magnet for the LHC," IEEE Trans. Magn., vol. 28, pp. 358-361, Mar. 1992.

[6] M. Bajko, P. Fessia, and D. Perini, "FEM computations concerning the effect of friction in two LHC main dipole structures," IEEE Trans. Appl. Superconduct., vol. 10, pp. 81-84, Mar. 2000.

[7] D. Dell'Orco, "Finite element analysis of the QC quadrupole for the superconducting supercollider,", LBL-29 600, Oct. 1989.

[8] M. Zaslawsky, "Nonlinear finite element analysis of the SSC superconductivity magnet: Including the effects of cooldown, operation, quench, preassembly loads and Lorentz forces," SSC-126, Apr. 1987.

[9] P. Thompson et al., "Mechanical properties of Isabelle superconducting coil," IEEE Trans. Nucl. Sci., vol. NS-28, pp. 3303-3305, June 1981.

[10] E. Acerbi and L. Rossi, "Mechanical, thermal and electrical measurements on material and components of the main coils of the Milan superconducting cyclotron," INFN/TC-88/16, Apr. 1988.

[11] F. W. Markley and J. S. Kerby, "Investigation of the mechanical properties of superconducting coils," in Supercollider 2, M. McAshan, Ed. New York: Plenum, 1990, pp. 753-763.

[12] I. R. Dixon, R. P. Walsh, W. D. Markiewicz, and C. A. Swenson, "Mechanical properties of epoxy impregnated superconducting solenoids," IEEE Trans. Magn., vol. 32, Aug. 1996.

[13] T. Saito et al., "Investigation of the mechanical and electrical properties of superconducting coils," in Supercollider 5, P. Hale, Ed. New York: Plenum, 1994, pp. 225-228.

[14] F. W. Markley, J. S. Kerby, B. Sizemore, C. Khoun, and T. King, "Investigation of the elastic modulus of SSC coils," in Supercollider 3, J. Nonte, Ed. New York: Plenum, 1991, pp. 165-173.

[15] N. I. Andreev, S. S. Kozub, V. V. Sytnik, A. T. Veshchikov, and A. V. Zlobin, "Mechanical properties of the coil for the UNK superconducting magnets," in IHEP 93-61, Protvino, 1993.

[16] Y. Chen, R. Jayakumar, and K. Yu, "Experimental Young modulus calculations," in Supercollider 5, P. Hale, Ed. New York: Plenum, 1994, pp. 181-184.

[17] F. Zerobin, M. Painer, and S. Eichberger, "Size and stiffness measurements on 9.5 m long LHC dipole coils," IEEE Trans. Magn., vol. 30, July 1994.

[18] R. E. Sims, "Tooling and process parameters improvements leading to improved dimensional control of the cured SSC dipole coils," in Supercollider 3, J. Nonte, Ed. New York: Plenum, 1991, pp. 57-74.

[19] C. A. Swenson, I. R. Dixon, and W. D. Markiewicz, "Measurements of thermal contraction properties for $\mathrm{NbTi}$ and $\mathrm{Nb}_{3} \mathrm{Sn}$ composites," IEEE Trans. Appl. Superconduct., vol. 7, pp. 408-411, June 1997. 
[20] K. P. Chow and G. A. Millos, "Measurements of modulus of elasticity and thermal contraction of epoxy impregnated Niobium-Tin and Niobium-Titanium composites," IEEE Trans. Appl. Superconduct., vol. 9, pp. 213-215, June 1999.

[21] R. B. Meuser, S. Caspi, and W. S. Gilbert, "Measured mechanical properties of superconducting coil materials and their influence on coil prestress," IEEE Trans. Magn., vol. 17, pp. 2320-2323, Sept. 1981.

[22] M. Reytier, A. Devred, M. Durante, C. Gourdin, and P. Vedrine, "Characterization of the thermo-mechanical behavior of insulated cable stacks representative of accelerator magnet coils," IEEE Trans. Appl. Superconduct., vol. 11, pp. 3066-3069, Mar. 2001

[23] C. Goodzeit, "Thermal contraction measurements," BNL-MDN-445-15, July 1992.

[24] V. N. Gladky, S. S. Kozub, A. T. Veshchikov, and U. Escher, "Thermal contraction of superconducting magnet materials," Cryogenics, vol. 35, no. 1, pp. 67-69, 1995

[25] P. Vedrine, B. Gallet, and C. Nouvel, "Measurement of thermo-mechanical properties of NiTi windings for accelerator magnet," IEEE Trans. Appl. Superconduct., vol. 9, pp. 236-239, June 1999.

[26] A. Devred et al., "About the mechanics of SSC dipole magnet prototypes," SSCL-Preprint-6, Nov. 1991.

[27] K. Artoos et al., "Design, manufacturing status, first results of the LHC main dipole final prototypes and step toward series manufacture," IEEE Trans. Appl. Superconduct., vol. 10, pp. 98-102, June 1999.

[28] C. Meuris, B. Baudouy, D. Leroy, and B. Szeless, "Heat transfer in electrical insulation of LHC cables cooled with superfluid helium," Cryogenics, vol. 39, pp. 921-931, Nov. 1999.

[29] G. Spigo, private communication, Jan. 2001.

[30] A. Devred, private communication, Jan. 2001.

[31] Y. S. Touloukian, R. K. Kirby, R. E. Taylor, and P. D. Desai, "Thermal expansion. Metallic elements and alloys," in Thermophysical Properties of Matter. New York: IFI/Plenum, vol. 12.

[32] N. Siegel, D. Tommasini, and Y. Vanenkov, "Design and use of capacitive force transducers for superconducting magnet models for the LHC," in Proc. 15th Int. Conf. Magnet Technol., L. Liangzhen, S. Guoliao, and Y. Luguang, Eds., Beijing, China, May 1998, 1997 CERN-LHC Project Rep. 173, p. 110

[33] N. Andreev et al., "Mechanical behavior of the short models of LHC main dipole magnets," CERN-LHC Project, Rep. 179, May 1998.

[34] D. R. Chichili, T. T. Arkan, J. P. Ozelis, and I. Terechkine, "Investigation of cable insulation and thermo-mechanical properties of epoxy impregnated $\mathrm{Nb}_{3}$ Sn composites," IEEE Trans. Appl. Superconduct., vol. 10, Mar. 2000.

[35] D. R. Chichili, T. T. Arkan, and I. Terechkine, "Investigation of cable insulation and mechanical properties of $\mathrm{Nb}_{3} \mathrm{Sn}$ composites," in FERMILAB Conf. 99/052, Apr. 1999.
Karine Couturier was born in Grenoble, France. She received the Engineer Diploma of the ENSEEG School (INPG-Grenoble) and the Ph.D. degree in materials science and engineering from INPG at Grenoble in 1995 and 1998, respectively.

She is currently responsible for material projects, especially mechanical testing, at CERN, Switzerland.

Paolo Ferracin was born in Carmagnola, Torino, Italy, on July 23, 1973. He received the M.S. degree in nuclear engineering from the Polytechnic of Turin, Italy, in 1998. He is currently pursuing the Ph.D. degree at the Polytechnic Institute of Turin.

He is a member of the Main Magnet and Superconductors (MMS) Group, CERN, Geneva, Switzerland.

Walter Scandale was born in Catanzaro, Italy, on November 5, 1944. He received the M.S. degree in physics in Rome where he also followed a School in Computing and Control Systems Engineering and worked in the Siemens Data as System Engineer.

Since November 1972, he has been an Applied Physicist at CERN. His research interests include accelerator physics and magnet technology.

Ezio Todesco was born in Bologna, Italy, on June 9, 1965. He received the M.S. degree in physics, in 1989, and the Ph.D. degree in mathematical physics, in 1994, at the Bologna State University.

Since 1998, he has been working at CERN on modeling and analyzing field quality in the superconducting dipoles of the Large Hadron Collider. He has been working on nonlinear dynamics of conservative systems, focusing on applications of perturbative theory to particle motion in accelerators, beam stability, and analysis of resonance phenomena.

Davide Tommasini received the M.S. degree in electrical engineering from the University of Genova, in 1986, and the Ph.D. degree from the Electrotechnical Institute of the University of Genova, Italy, in 1989.

From 1989 to 1994, he was leading the Storage Ring Magnets and Fast Magnets Group at Elettra, Trieste, Italy. His research interests include design and fabrication of classical and pulsed magnets and of high voltage-high current pulsed power supplies. Since 1994, he has been with CERN in Geneva, working for the LHC project in the field of superconducting magnets technologies. 